\title{
Period gene expression in relation to seasonality and circadian rhythms in the linden bug, Pyrrhocoris apterus (Heteroptera)
}

\author{
MAgDAlENa HODKOVÁ, ZdeŇKa SYROVÁ, DAVID DOLEŽEL and Ivo ŠAUMAN \\ Institute of Entomology, Academy of Sciences, České Budějovice, CZ-370 05 Czech Republic; e-mail: magda@entu.cas.cz
}

Key words. circadian clock, per mRNA, photoperiod, diapause, locomotor activity rhythms

\begin{abstract}
Wild females of Pyrrhocoris apterus exhibit seasonal changes in neuroendocrine activity and, consequently, reproduction. Long days (18 h light/6 h dark) (LD) stimulate reproduction, whereas short days (12 h light/12 h dark) (SD) induce reproductive arrest (diapause). This study reveals how photoperiod influences the expression of the circadian clock gene, period (per) in the insect's head. There is only a weak diurnal rhythm in per mRNA expression under LD and SD. However, levels of per mRNA are consistently higher (up to 10-fold) under SD than under LD. The influence of photoperiod on per gene expression is linked to a developmental output (diapause vs. reproduction); mutant females, reproducing under both LD and SD, show low per mRNA levels under both photoperiodic conditions. Thus, the magnitude of per gene expression may be important to the translation of photoperiodic signals into a hormonal message. Levels of per mRNA are related to properties of locomotor activity rhythms. Low per mRNA levels (displayed by wild females in LD and mutant females in both LD and SD) are associated with long free-running periods $(\tau \sim 26-27 \mathrm{~h})$ and late peaks of activity $\left(\psi_{\mathrm{R}, \mathrm{L}} \sim 10-12 \mathrm{~h}\right)$, whereas high per mRNA levels coincide with short free-running periods $(\tau \sim 24 \mathrm{~h})$ and early peaks of activity $\left(\psi_{\mathrm{R}, \mathrm{L}} \sim 4-6 \mathrm{~h}\right)$. Overall, the data provide a background for a molecular approach to the longstanding question about the role of the circadian system in insect photoperiodism.
\end{abstract}

\section{INTRODUCTION}

It is generally accepted that biological timing depends not only on the generation of circadian rhythms, but also on the measurement of time intervals (day length/length of night). While the expression of circadian output determines the circadian activities of the organism, the interval timing results in seasonal changes in the developmental pathways. The relationship between these two phenomena is still largely unresolved. Formal properties of photoperiodic responses indicate that photoperiodic time measurement is a function of the circadian system (Saunders, 1998; Vaz Nunes \& Saunders, 1999). Recently, Veerman (2001) suggested that downstream events, such as synthesis of a clock substrate, are under circadian control in insects and mites. While our understanding of molecular mechanisms of circadian rhythms has greatly advanced, particularly due to the enormous power of Drosophila melanogaster genetics (Schotland \& Sehgal, 2001), molecular events, from photoreception through time measurement and accumulation of photoperiodic "information" to neuroendocrine activity, controlling the onset of diapause or continuation of development/reproduction are still unknown.

Although the period gene (per) has been shown to form a central part of the circadian clock of insects as well as vertebrates (Dunlap, 1999; Reppert \& Weaver, 2000; Schotland \& Seghal, 2001), its role in the photoperiodic regulation of development is uncertain. Saunders (1990) concluded that the per gene in not causally involved in the photoperiodic induction of ovarian diapause in $D$. melanogaster; flies in which the per locus was missing $\left(p e r^{0}\right)$ were still able to discriminate between diapauseinducing short days and diapause-averting long days, although the critical daylength was altered. Studies of latitudinal clines in photoperiodic responses show only weak correlation between critical daylength and period $(\tau)$ of eclosion rhythm (e.g. Pittendrigh et al., 1984 - D. auraria; Lankinen, 1986 - D. litoralis) or $\tau$ of the NandaHamner rhythm (Vaz Nunes et al., 1990 - Tetranychus urticae). Furthermore, Nanda-Hamner experiments indicate that $\tau$ for the photoperiodic oscillator differs from that for locomotor rhythm in Calliphora vicina (Saunders, 1987a; Hong \& Saunders, 1998). The above examples indicate that overt behavioural rhythmicity and photoperiodic timing involve separate circadian pacemakers with different characteristics (Saunders, 2001). On the other hand, day length affects the expression of per mRNA (Majercak et al., 1999), as well as the $\tau$ of locomotor rhythm in D. melanogaster (Tomioka et al., 1997). The effect of day length is mediated through the photosensitive timeless protein (Majercak et al., 1999), and the timeless locus is thought to be causally involved in the photoperiodic induction of larval diapause in another drosophilid, Chymomyza costata (Kostal \& Shimada, 2001; Pavelka et al., 2003). These results indicate that the circadian clock governing overt rhythms and mechanisms decoding photoperiodic time may share common molecular components.

A heteropteran insect, Pyrrhocoris apterus, exhibits an adult diapause controlled by photoperiod; long days stimulate the activity of the corpus allatum (CA) and reproduction, whereas short days are inhibitory. Effect of daylength on the CA is mediated through the pars intercerebralis (PI) of the brain (Hodková, 1976) and per mRNA is expressed in neurosecretory cells of the PI (Syrová et al., 2001). Although it is unlikely that a differ- 
TABLE 1. Characteristics of locomotor activity rhythms in females of $P$. apterus under different photoperiods.

\begin{tabular}{|c|c|c|c|c|c|c|}
\hline Insects & Photoperiod & Food conditions & Replicate & $\begin{array}{l}\text { Free-running } \\
\text { period }(\tau)(h)^{1)}\end{array}$ & $\begin{array}{c}\text { Activity peak } \\
\left(\psi_{\mathrm{R}, \mathrm{L}}\right)(\mathrm{h})^{2)}\end{array}$ & $\begin{array}{c}\text { Activity level } \\
\text { (mesor) }\end{array}$ \\
\hline \multirow{6}{*}{$\begin{array}{l}\text { Photoperiodically } \\
\text { sensitive }\end{array}$} & \multirow{3}{*}{ long } & feeding & 1 & $26.4 * *$ & $10.6^{*}$ & 5.5 \\
\hline & & feeding & 2 & $27.2^{*}$ & $12.6 * *$ & 6.2 \\
\hline & & starving & 3 & $26.4 * *$ & $10.2^{*}$ & 6.4 \\
\hline & \multirow{3}{*}{ short } & feeding & 1 & $?$ & $8.1^{*}$ & 0.6 \\
\hline & & feeding & 2 & $?$ & $5.6^{*}$ & 2.7 \\
\hline & & starving & 3 & $24.2^{*}$ & $3.8 *$ & 1.9 \\
\hline \multirow{5}{*}{$\begin{array}{l}\text { Photoperiodically } \\
\text { insensitive }\end{array}$} & \multirow{2}{*}{ long } & feeding & 1 & $27.6^{* *}$ & $12.8 * *$ & 6.9 \\
\hline & & starving & 2 & $26.0^{* *}$ & $10.8^{*}$ & 11.3 \\
\hline & \multirow{3}{*}{ short } & feeding & 1 & $26.0^{* *}$ & $10.0^{*}$ & 9.8 \\
\hline & & feeding & 2 & $27.0^{* *}$ & $12.6^{*}$ & 15.9 \\
\hline & & starving & 3 & $26.2 * *$ & $11.2 *$ & 8.8 \\
\hline
\end{tabular}

$\tau$ and $\psi_{\mathrm{R}, \mathrm{L}}$ were calculated for 5-8 cycles, mesor was calculated for $10-13$ cycles.

1)**p $<0.005-0.01,{ }^{*} \mathrm{p}<0.05$

2)**excellent, * good

ence in any single clock component, such as per mRNA level, is responsible for decoding photoperiodic time, it may reflect an underlying difference in the circadian structure. We have investigated, using an RNase protection assay, how day length affects the expression of per mRNA in the insect's head. A potential relationship of per mRNA levels to developmental mode (diapause vs. reproduction) and properties of circadian rhythms in locomotor activity have been estimated using two laboratory strains of $P$. apterus differing in their diapause photoresponsiveness: wild-type insects, reproducing under long days and entering diapause under short days, and mutant insects, reproducing under both long day and short day conditions.

\section{MATERIAL AND METHODS}

\section{Insects}

Wild-type and mutant strains of P. apterus (L.) (Heteroptera) used in this study originated from adults collected from the field near České Budějovice, Czech Republic. Insects were reared at $26 \pm 1^{\circ} \mathrm{C}$ on linden seed and water ad libitum. The wild-type strain (referred to as photoperiodically sensitive insects) was maintained under diapause-preventing long days (LD) (18 h light / $6 \mathrm{~h}$ dark). A selected non-diapause strain (referred to as photoperiodically insensitive) was maintained under short days (SD) (12 h light / $12 \mathrm{~h}$ dark). Experimental insects were reared from the egg under either LD or SD. In addition to feeding females, locomotor activity rhythms were monitored in starving females, provided with only water, to discriminate a potential masking effect of feeding and oviposition behaviour. In $P$. apterus, feeding is essential for ovarian maturation. Only feeding females were used for RNase protection assay analysis.

\section{Locomotor activity rhythms}

Locomotor activity was monitored by placing individual females in Petri dishes and using a recording device comprised of an infrared beam passing horizontally through the dish to a phototransistor. Activity was monitored in $12 \mathrm{~min}$ bins as the number of interruptions of the infrared light beam by moving insects and the data were recorded by computer. Monitoring was performed at $26 \pm 1{ }^{\circ} \mathrm{C}$. Light was provided by a fluorescent lamp ( 9 watts) controlled by a $24-\mathrm{h}$ timer. Females aged 1-2 days were exposed to 5-8 light cycles before being transferred to constant darkness (DD) for 5-8 days. The average rhythm characteristics were calculated by pooling data for 5-8 females. Three independent measurements (two with feeding females, one with starving females) were analysed. The phase difference between the time of peak activity and light on $\left(\psi_{\mathrm{RL}}\right)$ was calculated using an acrophase program, fitting a cosine wave to the data (Refinetti, 2000). The free-running period $(\tau)$ under DD was determined by chi-square periodogram analysis (Refinetti, 2000). The activity level was calculated as the arithmetic mean of all values (mesor) in the light cycles and DD. In the figures, the mean of 5 values $(5 \times 12 \mathrm{~min}=1 \mathrm{~h})$ was calculated for every hour.

\section{RNase protection assay}

Heads (without antennae and rostrum) were cut every $4 \mathrm{~h}$ around the clock and immediately placed on dry ice, and kept at $-85^{\circ} \mathrm{C}$ until analysis. For each time point 25 heads were used for total RNA isolation. Total RNA was extracted using the RNA Blue isolation system (Top-Bio). [ $\left.{ }^{32} \mathrm{P}\right]$ UTP-labelled per cRNA antisense and sense probes were generated by subcloning PCRamplified cDNA fragments into pBluescript, followed by in vitro transcription driven from T3 and T7 RNA polymerase promoters, respectively. An antisense RP49 reference probe cloned from $P$. apterus (unpublished data) was included in each RNase protection reaction as an internal control for the amount of RNA loaded in each lane. To equalize the signal intensity of the per and RP49 bands on the gel, the specific activity of the RP49 probe was reduced by diluting the $\left[{ }^{32} \mathrm{P}\right] \mathrm{UTP} 1: 750$ with nonradioactive UTP in the in vitro transcription reaction. RNase protection assays were performed using a RPA III kit (Ambion) according to the supplied protocol. Quantification was performed on a Storm PhosphorImager (Molecular Dynamics) and ImageQuant software (Molecular Dynamics). Each protection assay was performed at least three times with similar results. The results were replicated with at least two sets of animals for each experiment.

\section{RESULTS}

\section{Effect of photoperiod on locomotor rhythms Photoperiodically sensitive insects}

Females showed significant diurnal locomotor activity rhythms under both LD and SD conditions with periods close to $24 \mathrm{~h}$, similar to that of the light cycle. The peak of activity in females held in LD was in late afternoon in all experimental groups $\left(\psi_{\mathrm{R}, \mathrm{L}}=10.2-12.6 \mathrm{~h}\right.$ ) (Table 1, 

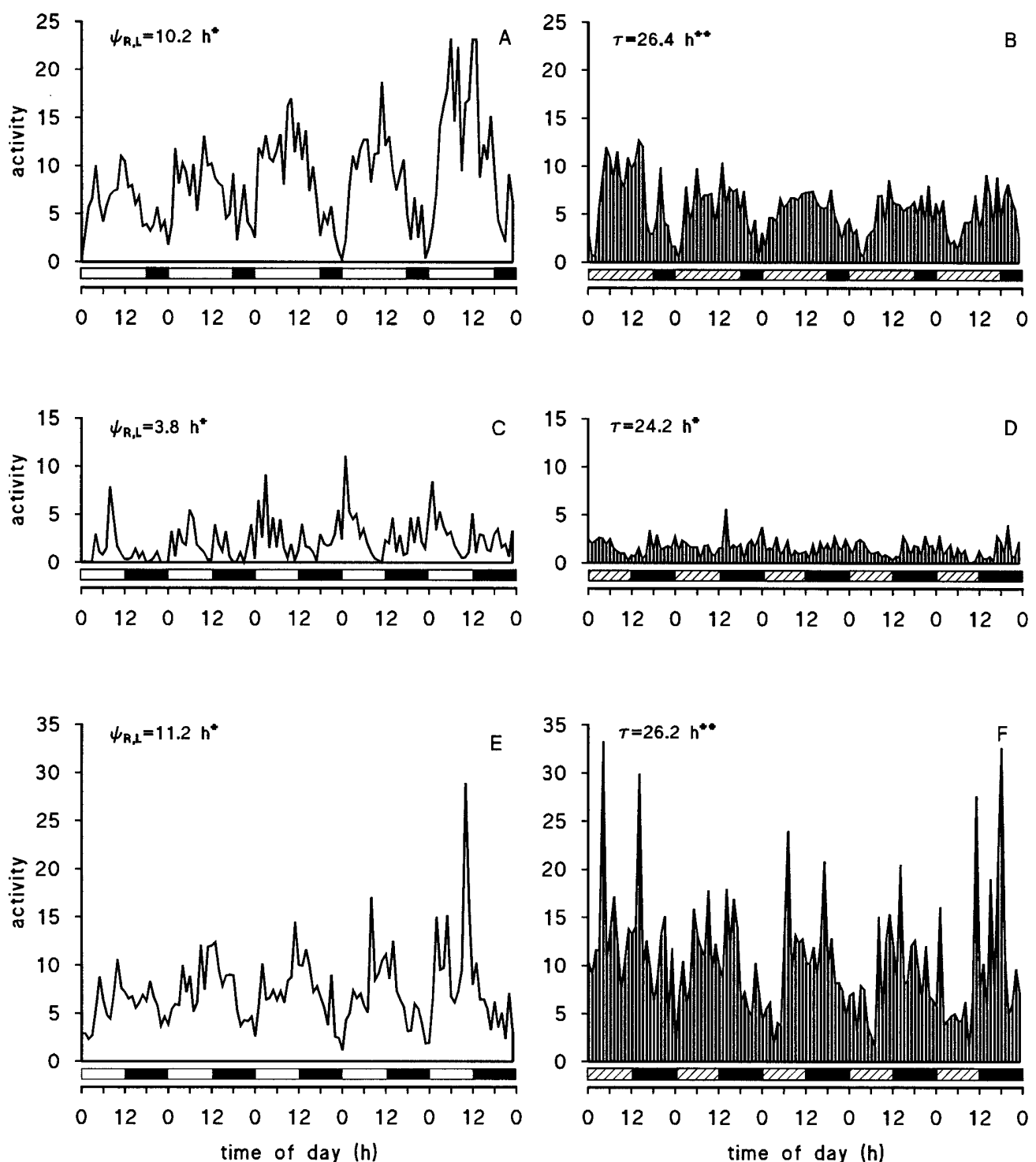

Fig. 1. Diurnal and circadian patterns of locomotor activity in starving females of $P$. apterus under different photoperiods. Photoperiodically sensitive females were held in long day (A), constant darkness after long day entrainment (B), short day (C), constant darkness after short day (D). Photoperiodically insensitive females were held in short day (E), constant darkness after short day (F). White and black horizontal bars represent times in daily cycles when the lights were either on or off, respectively. Hatched bars represent subjective day. For other explanations see Table 1.

Fig. 1A, 2A). Under DD, the rhythm free ran with a period longer than $26 \mathrm{~h}(\tau=26.4-27.2 \mathrm{~h})$ (Table 1, Fig. 1B). Females held at SD showed a considerable difference in rhythm properties relative to SD females. The activity level under SD was about one-third of that under LD (Table 1, Fig. 1A-D, 2A,B). Furthermore, the peak of activity under SD was several hours earlier relative to $\mathrm{LD}$ $\left(\psi_{\mathrm{R}, \mathrm{L}}=3.8-8.1 \mathrm{~h}\right)$ (Table 1, Fig. 1C, 2B). Feeding SD females appeared arrhythmic under DD (Table 1), probably due to a low locomotor activity associated with a masking effect of feeding activity. In starving SD females, however, the free-running period was shorter by about two hours compared to starving LD females $(\tau=$ 24.2 h) (Table 1, Fig. 1D).

\section{Photoperiodically insensitive insects}

Rhythm properties in photoperiodically insensitive females showed fewer differences between LD and SD compared to photoperiodically sensitive females. In both LD and SD females, the peak of activity occurred in the late afternoon $\left(\psi_{\mathrm{R}, \mathrm{L}}=10.8-12.8 \mathrm{~h}\right.$ for $\mathrm{LD}, 10.0-12.6 \mathrm{~h}$ for SD) (Table 1, Fig. 1E). The free-running period was $26 \mathrm{~h}$ or longer under DD in both LD females $(\tau=26.0-27.6)$ (Table 1) and SD females $(\tau=26.0-27.0 \mathrm{~h}$ ) (Table 1, Fig. $1 \mathrm{~F})$. Under both LD and SD, the rhythm assumed a period close to the 24-h period of the light cycle. The activity level was high under both photoperiods; it tended to be higher under SD than under LD in feeding females, while in starving females an opposite trend was observed (Table $1)$. It is noteworthy that the rhythm characteristics ( $\tau$, $\psi_{\mathrm{R}, \mathrm{L}}$ ) in photoperiodically insensitive females held in SD 

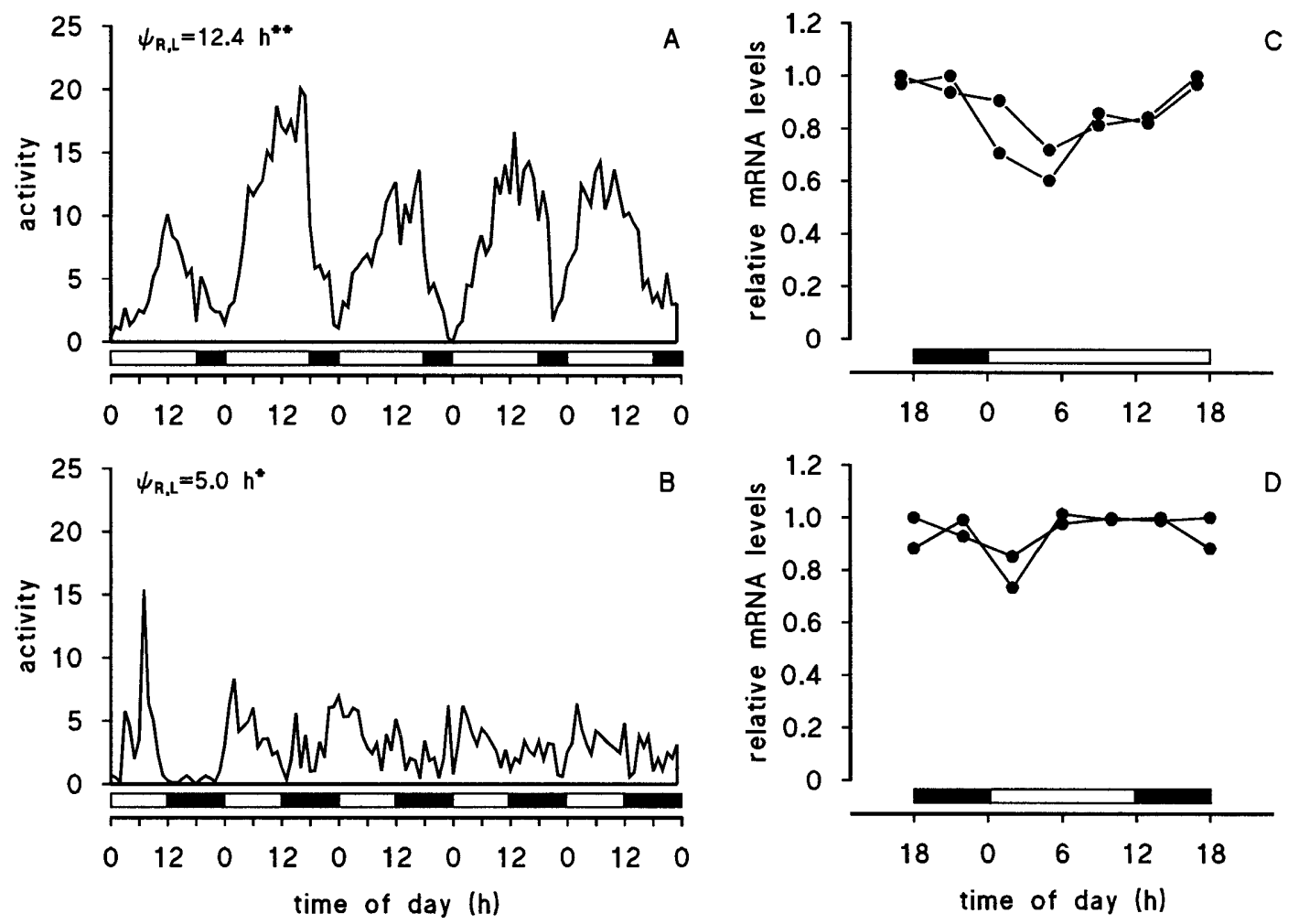

Fig. 2. Diurnal rhythms in locomotor activity and relative levels of per mRNA in feeding females of $P$. apterus under different photoperiods. Photoperiodically sensitive females were held in long day $(A, C)$ or short day $(C, D)$. Relative per mRNA levels refer to per/RP49 ratio. For other explanations see Fig. 1 and Table 1.

were similar to those for photoperiodically sensitive females under LD. The overall activity tended to be higher in photoperiodically insensitive relative to sensitive females (Table 1).

\section{Effect of photoperiod on per mRNA expression}

Temporal changes in per mRNA levels in heads of photoperiodically sensitive females revealed a weak diurnal rhythm under LD, with a peak of expression at Zeitgeber time (ZT) 17-21 and minimum expression at ZT 5 (Fig. $2 \mathrm{C}$ ). Under SD, there was no distinct oscillation in per mRNA expression. Levels of per mRNA were consistently high, with the exception of a slight decrease at ZT 2 (Fig. 2D). A delayed increase in the per mRNA level under $\mathrm{LD}$ relative to $\mathrm{SD}$ (Fig. 2 C,D) corresponds to a delayed peak in the locomotor rhythm under LD (Table 1, Fig. 2 A,B). The most striking difference between LD and SD females was a considerably higher expression of per

TABLE 2. Relative per mRNA levels in feeding females of $P$. apterus under different photoperiods.

\begin{tabular}{lccc}
\hline Insects & Photoperiod & Zeitgeber time (h) & per/RP49 \\
\hline \multirow{3}{*}{$\begin{array}{lcc}\text { Photoperiodically } \\
\text { sensitive }\end{array}$} & \multirow{2}{*}{ long } & 5 & 0.08 \\
& & 17 & 0.20 \\
& short & 6 & 1.00 \\
Photoperiodically & \multirow{2}{*}{ long } & 5 & 0.87 \\
insensitive & & 17 & 0.13 \\
& \multirow{2}{*}{ short } & 6 & 0.22 \\
& & 18 & 0.21 \\
\hline
\end{tabular}

mRNA under SD. Levels of per mRNA were consistently higher under SD (up to 10-fold) than under LD (Fig. $3 \mathrm{~A}$, B).

To see whether photoperiod influences the magnitude of per gene expression in photoperiodically insensitive females, per mRNA levels were compared between LD and SD at two time points (Table 2 ). These preliminary data show that per mRNA levels detected in photoperiodically insensitive females were low under both SD and LD and similar to those found in photoperiodically sensitive females held in LD. Low per mRNA levels in the three groups of females coincided with a higher activity level, later activity peak, and longer free-running period of their locomotor rhythms compared to photoperiodically sensitive females held in SD, which showed high per mRNA levels (see above).

\section{DISCUSSION}

The conventional view that the circadian system is involved in the transduction of photoperiodic signals into a developmental output (diapause vs. continued development/reproduction) (see Introduction) implies that molecular components of the circadian system somehow respond to alterations in photoperiod. Here we show how photoperiod regulates the expression of the essential circadian clock gene, period, in heads of $P$. apterus females. Photoperiod is translated into the magnitude of per gene expression, with a considerably higher expression under SD than under LD. However, the strong up-regulation of per gene expression under SD occurs only in wild-type 
A

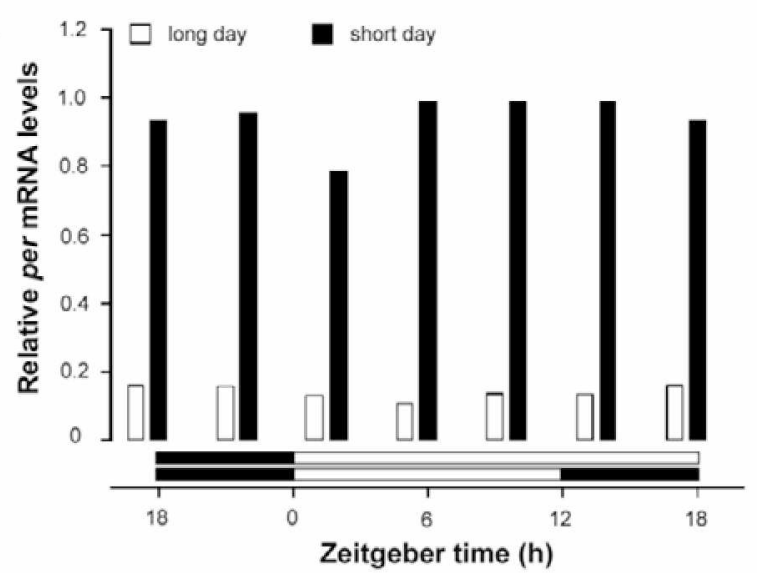

B

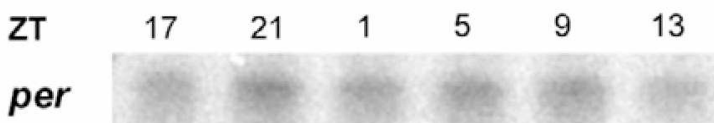

RP49
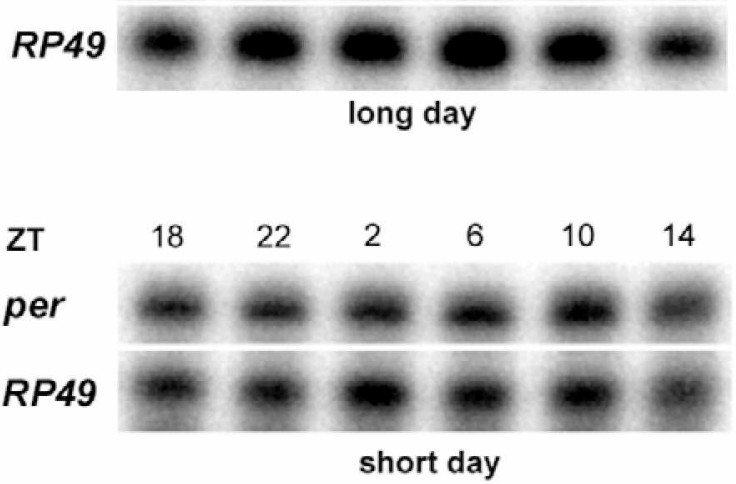

Fig. 3. Effect of photoperiod on per mRNA levels in feeding females of $P$. apterus. A - relative per mRNA levels were compared between long day and short day females sensitive to photoperiod. Values represent means of two determinations; B RNAs were visualised by autoradiography. For other explanations see Fig. 1 and 2.

(photoperiodically sensitive) females that enter diapause under SD. Mutant (photoperiodically insensitive) females, reproducing under both $\mathrm{LD}$ and $\mathrm{SD}$, fail to increase per mRNA levels under SD (Table 3). Because per mRNA levels are linked to both photoperiod and developmental output, the magnitude of per gene expression may be important to the transduction of photoperiodic signals into a hormonal message. This view is supported by findings that per mRNA is expressed in the neurosecretory cells of the PI of the brain (Syrová et al., 2001) that are implicated in the translation of photoperiod into neurohormonal signals (Hodková, 1976). It is not clear, however, whether the per gene is a component of the photoperiodic clock or its target. The photoperiodic response of $P$. apterus presents a few unusual features. This species appears to "measure" day length rather than length of night and Nanda-Hamner results indicate an extremely short free-running period $(16 \mathrm{~h})$ of the constituent oscillators (Saunders, 1987b). On the other hand, free-running periods of locomotor rhythms are longer than $24 \mathrm{~h}$ (Table 1 , Figs 1,2). However, there is only a weak diurnal rhythm of per mRNA under SD and LD (Fig. 2). The difference in the level of per mRNA between SD and LD suggests, perhaps, that the per gene plays no central role as a clock component, but might be on the output pathway to diapause/reproduction. Although there may be no causal relationship between the per gene itself and photoperiodic regulation of diapause, as is suggested for D. melanogaster (Saunders, 1990), it is likely that the photoperiodic regulation of per gene expression, as revealed in $P$. apterus, reflects responses of other molecular components of the circadian system to photoperiod. It will be important to study how photoperiod affects other circadian clock-related genes, particularly in the view of a recent finding that the photoperiodic insensitivity in a drosophilid fly, C. costata, may be caused by an inability to transcribe the timeless (tim) gene (Pavelka et al., 2003). A higher (about twice) peak level of tim mRNA under short daylength relative to long daylength was demonstrated in adult heads of the flesh fly, Sarcophaga crassipalpis (Goto \& Denlinger, 2002). It is not clear, however, whether the level of tim gene expression in adults has any relation to pupal diapause in this species. The molecular basis for the absence of diapause photoresponsiveness in the photoperiodically insensitive strain of $P$. apterus is not known. If the per gene is part of a photoperiodic transduction chain, the block to diapause photoresponsiveness may be expected upstream from the per mRNA expression. Diapause photoresponsiveness also disappears during diapause development in wild-type females of $P$. apterus (Hodek, 1971). It will be interesting to study how these ontogenetic changes in photoperiodic response affect per mRNA expression.

TABLE 3. Per mRNA levels and activity rhythm properties in relation to photoperiod and developmental program (summary).

\begin{tabular}{lcccc}
\hline & \multicolumn{2}{c}{ Photoperiodically sensitive insects } & Photoperiodically insensitive insects \\
\hline Photoperiod & long & short & long & short \\
Developmental program & reproduction & diapause & reproduction & reproduction \\
per mRNA level & low & high & low & low \\
Free-running period & long & short & long & long \\
Activity peak & late & early & late & late \\
Activity level & high & low & high & high \\
\hline
\end{tabular}

For details see Table 1,2, Fig. 1-3, and text. 
The period and timeless genes seem to be essential components of the circadian clock underlying overt rhythmicity in D. melanogaster (Dunlap, 1999; Sauman \& Hashimi, 1999; Schotland \& Sehgal, 2001). In P. apterus, diurnal rhythms in the relative abundance of per mRNA show no robust peaks (particularly under SD) that would indicate a clear relation to locomotor activity rhythms. On the other hand, the magnitude of per gene expression seems to be related to the free-running period of the activity rhythm. It was shown in $D$. melanogaster that the free-running period for circadian rhythms is inversely related to the log of per mRNA titre (Baylies et al., 1987). Similarly, in P. apterus, high levels of per mRNA were associated with a short free-running period $(\tau \sim 24 \mathrm{~h})$, while low levels of per mRNA under LD coincided with a long free-running period ( $\tau \sim 26-27 \mathrm{~h}$ ) of the activity rhythm (Table 3 ). The mechanism by which per mRNA levels are regulated by photoperiod is not known. The rhythm assumed a 24- hour period (i.e. the period of light cycle) with a phase angle difference $\left(\psi_{R, L}\right)$ depending on $\tau$ (the longer the $\tau$, the later the peak - Table 1), in accordance with general rules of the entrainment of circadian rhythms (Pittendrigh, 1981), in both wild and mutant females of $P$. apterus. Therefore, the failure of mutant females to increase per mRNA levels under SD cannot be explained by decoupling of circadian system from the entraining effect of light. Conversely, it may be assumed that the influence of photoperiod on per mRNA levels in wild females is not executed through an entrainment pathway. The downstream pathway from the magnitude of per gene expression to the free-running period of activity rhythm is not known either. Properties of locomotor activity rhythms $\left(\tau, \psi_{\mathrm{RL}}\right.$, activity level) were related to a developmental program determined by photoperiod (Table 3). It is unlikely, however, that final outputs, such as vitellogenesis and oviposition, are responsible for the difference in rhythm properties between $\mathrm{LD}$ and SD; the difference was also found in starving females, although vitellogenesis was prevented (Fig. 1A-D). The CA is inhibited from the PI in both SD females and starving LD females of $P$. apterus, but the inhibition caused by SD is qualitatively different from that caused by starvation (Hodková et al., 2001). Findings that removing the neurosecretory cells of the PI influences properties of locomotor rhythms (unpublished data) and mating rhythms (Hodková, 1994) indicate a neurohormonal regulation of the circadian rhythms in $P$. apterus. A juvenile hormone analogue (methoprene) affected the $\tau$ of the eclosion rhythm in the southwestern corn borer, Diatraea grandiosella (Yin et al., 1987). There is evidence that light cycles perceived during development affect properties of circadian rhythms, particularly their free-running period (e.g. Barrett \& Page, 1989; Tomioka et al., 1997; Wattari, 2002), but the physiological and molecular mechanisms of these alterations are still largely unknown.

In addition to the PI, per expressing cells were found in the compound eyes (Syrová et al., 2001), where the circadian pacemaker for locomotor rhythmicity was identified in P. apterus (Hodková, 1999). It is possible that similar molecular components are used by different tissues for different functions: Translation of day length into a neuroendocrine message by the PI and entrainment of the circadian rhythms by the compound eyes. In a mammal, the Syrian hamster, photoperiod affects the amplitude of an early-response gene, Perl, expression in the pars tuberalis that is implicated in decoding day length. In contrast, photoperiod has no effect on the gene expression in the suprachiasmatic nucleus that is the site of the circadian pacemaker (Messager et al., 1999). Studies are still needed to determine how photoperiod affects clock gene expression in individual per-expressing tissues in $P$. apterus.

ACKNOWLEDGEMENTS. We thank to Jana Mikešová for technical assistance. The study was supported by the Grant Agency of the Czech Republic (Grants 206/02/0900 and 204/01/0404) and by the Academy of Sciences of the Czech Republic (Project Z5007907).

\section{REFERENCES}

BarRetT K.P. \& PAGe T.L. 1989: Effects of light on circadian pacemaker development. I. The freerunning period. J. Comp. Physiol. A 165: 41-49.

Baylies M.K., Bargiello T.A., Jackson F.R. \& Young M.W. 1987: Changes in abundance or structure of the per gene product can alter periodicity of the Drosophila clock. Nature 326: 390-392.

DunLAP J.C. 1999: Molecular bases for circadian clock. Cell 96: 271-290.

Goto S.G. \& Denlinger D.L. 2002: Short-day and long-day expression patterns of genes involved in the flesh fly clock mechanism: period, timeless, cycle and cryptochrome. $J$. Insect Physiol. 48: 803-816.

Hong S.-F. \& SAUnders D.S. 1998: Internal desynchronisation of the circadian locomotor rhythm in the blow fly, Calliphora vicina, as evidence for the involvement of a complex pacemaker. Biol. Rhythm Res. 29: 387-396.

Hodek I. 1971: Termination of adult diapause in Pyrrhocoris apterus (Heteroptera: Pyrrhocoridae) in the field. Entomol. Exp. Appl. 14: 212-222.

HodKová M. 1976: Nervous inhibition of corpora allata by photoperiod in Pyrrhocoris apterus. Nature 263: 521-523.

HoDKoví M. 1994: Photoperiodic regulation of mating behaviour in the linden bug, Pyrrhocoris apterus is mediated by a brain inhibitory factor. Experientia 50: 742-744.

HodKová M. 1999: Regulation of diapause and reproduction in Pyrrhocoris apterus (L.) (Heteroptera) - Neuroendocrine outputs (mini-review). Entomol. Sci. 2: 563-566.

Hodková M., OKudA T. \& WAGNer R.M. 2001: Regulation of corpora allata in females of Pyrrhocoris apterus (Heteroptera) (a mini-review). In vitro Cell. Dev. Biol.-Animal 37: 560-563.

Kostal V. \& Shimada K. 2001: Malfunction of circadian clock in the non-photoperiodic diapause mutants of the drosophilid fly, Chymomyza costata. J. Insect Physiol. 47: 1269-1274.

LANKINEN P. 1986: Geographical variation in circadian eclosion rhythm and photoperiodic adult diapause in Drosophila littoralis. J. Comp. Physiol. A 159: 123-142.

Majercak J., Sidote D., Hardin P.E. \& Ederry I. 1999: How a circadian clock adapts to seasonal decreases in temperature and day length. Neuron 24: 219-230.

Messager S., Ross A.W., Barrett P. \& Morgan P.J. 1999: Decoding photoperiodic time through Per 1 and ICER gene amplitude. Proc. Natl. Acad. Sci. USA 96: 9938-9943. 
Pavelka J., Shimada K. \& Kostal V. 2003: Timeless: A link between fly's circadian and photoperiodic clocks? Eur. J. Entomol. 100.

PitTENDRIGH C.S. 1981: Circadian systems: entrainment. In Aschoff J. (ed.): Biological Rhythms. Handbook of Behavioral Neurobiology 4, Plenum Press, New York, pp. 95-124.

Pittendrigh C.S., Elliot J. \& Takamura T. 1984: The circadian component in photoperiodic induction. In: Porter R. \& Collins G.M. (eds): Photoperiodic Regulation of Insect and Molluscan Hormones. Ciba Foundation Symposium No. 104. Pitman, London, pp. 26-47.

Refinetti R. 2000: Circadian Physiology. CRC Press, Boca Raton, $184 \mathrm{pp}$.

REPPERT S.M. \& WeAVER D.R. 2000: Comparing clockworks: mouse versus fly. J. Biol. Rhythms 15: 357-364.

SAUMAN I. \& Hashimi H. 1999: Insect clocks: what are they telling us besides time? Entomol. Sci. 2: 589-596.

SAUNDERS D.S. 1987a: Maternal influence on the incidence and duration of larval diapause in Calliphora vicina. Physiol. Entomol. 12: 331-338.

SAUNDERS D.S. 1987b: Insect photoperiodism: the linden bug, Pyrrhocoris apterus, a species that measures daylength rather than nightlength. Experientia 43: 935-937.

SAUNDERS D.S. 1990: The circadian basis of ovarian diapause regulation in Drosophila melanogaster: Is the period gene causally involved in photoperiodic time measurement? $J$. Biol. Rhythms 5: 315-331.

SAUNDERS D.S. 1998: Insect circadian rhythms and photoperiodism. Invertebr. Neurosci. 3: 155-164.

SAUNDERS D.S. 2001: Geographical strains and selection for the diapause trait in Calliphora vicina. In: Denlinger D.L., Giebultowicz J. \& Saunders D.S. (eds): Insect Timing: Circadian
Rhythmicity to Seasonality. Elsevier, Amsterdam, pp. 113-121.

Schotlant A. \& Semgal A. 2001: Molecular control of Drosophila circadian rhythms. In: Denlinger D.L., Giebultowicz J. \& Saunders D.S. (eds): Insect Timing: Circadian Rhythmicity to Seasonality. Elsevier, Amsterdam, pp. 15-30.

Syrová Z., Hodková M., Doležel D. \& Sauman I. 2001: Identification of putative circadian clock cells in a heteropteran insect, Pyrrhocoris apterus. Abstr. Cells III. Česke Budejovice.

Tomioka K., Uwozumi K. \& Matsumoto N. 1997: Light cycles given during development affect freerunning period of circadian locomotor rhythm of period mutants in Drosophila melanogaster. J. Insect Physiol. 43: 297-305.

VAz Nunes M., Koveos D.S. \& Veerman A. 1990: Geographical variation in photoperiodic induction in the spider mite (Tetranychus urticae): A causal relation between critical nightlength and circadian period ? J. Biol. Rhythms. 5: 47-57.

Vaz Nunes M. \& Saunders D.S. 1999: Photoperiodic time measurement in insects: A review of clock models. J. Biol. Rhythms 14: 84-104.

VeerMan A. 2001: Photoperiodic time measurement in insects and mites: a critical evaluation of the oscillator-clock hypothesis. J. Insect Physiol. 47: 1097-1109.

WATTARI Y. 2002: Comparison of the circadian eclosion rhythm between non-diapause and diapause pupae in the onion fly, Delia antiqua. J. Insect Physiol. 48: 83-89.

YN C.-M., TAKEDA M. \& WANG Z.-S. 1987: A juvenile hormone analogue, methoprene as a circadian and developmental modulator in Diatraea grandiosella (Pyralidae): J. Insect Physiol. 33: 95-102.

Received December 10, 2002; revised February 10, 2003; accepted April 3, 2003 